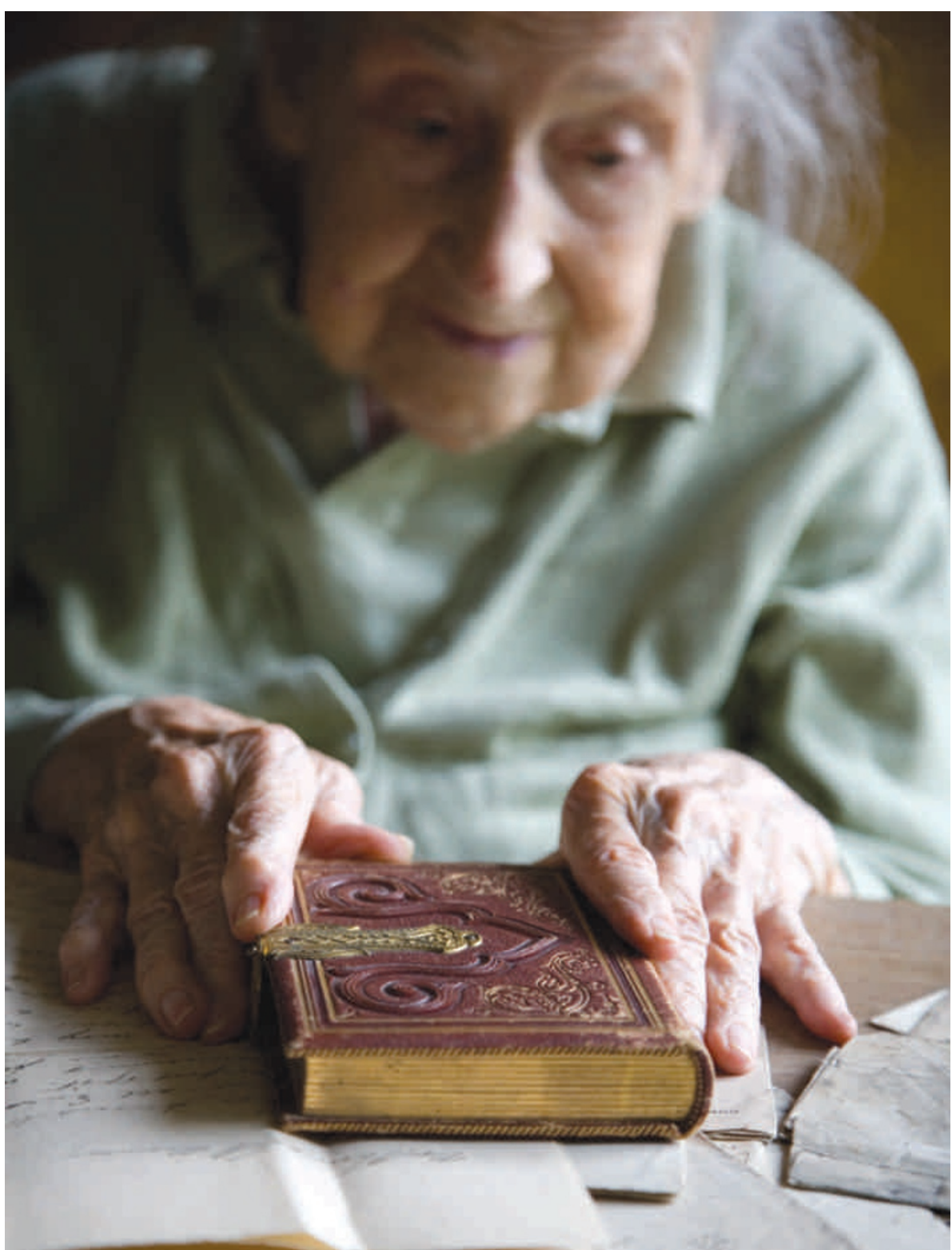

Elderly people often retain existing memories but may need help remembering new things.

\title{
COGNITION
}

\section{The brain's decline}

\section{Treating cognitive problems common in elderly people requires a deeper understanding of how a healthy brain ages.}

\section{BY ALISON ABBOTT}

wedish brothers Jonas and Robert af Jochnick became wealthy philanthropists after $\checkmark$ their direct-selling company, Oriflame Cosmetics, found success peddling dreams of ever-youthful complexions to post-communist Eastern Europe.

The brothers are now in their 70s, an age where dreams of youthful skin have morphed ambitious study is unusual in providing a wide range of tests on the same subjects over the coming years, with the aim of understanding how cognition declines as the brain ages - and whether we can do anything about it.

Bäckman's goal is to understand ordinary, non-disease-influenced ageing. "We all want to know what's normal," says Cheryl Grady, who studies ageing brains at the Rotman Research Institute in Toronto, Canada, "both as a reference for dementia research, and as a guide for any future interventions."

Given the concern in most developed countries about their ageing populations and the problem of dementia, it is surprising how little we know about healthy brain ageing. There are only a few things that researchers can agree on. First, after the age of 60, nearly everyone will start to experience some decline in cognitive skills, most noticeably in memory, and this decline will be accompanied by a change in brain structure. Second, aerobic exercise slows or delays this mental slippage.

The realm of uncertainty is much larger. Researchers still don't understand the mechanisms underlying the decline or the order of events. They can't explain why some people manage to stay cogent and alert well into their 80 s, whereas others become slow-witted and forgetful in their 60s. They don't even know whether Alzheimer's disease is an abnormal pathological condition or simply an acceleration of normal ageing. And no one knows of any drugs that can help those who lose cognitive function as they age, or whether brain training programmes really help.

\section{MEMORY FAILURE}

The COBRA study is recruiting healthy subjects aged 63-65 and aims to follow them for at least a decade. It will carry out various tests, including structural and functional imaging to see which parts of the brain are active on any given task, and how its volume changes over time. Diffusion tensor imaging will visualize the integrity of white matter - the long bands of neuronal axons that transmit signals between different brain regions. And positron-emission tomography (PET) will assess dopamine levels - this is rarely used, says Bäckman, because a single PET scan can cost several thousand dollars. In addition, subjects will carry out a wide range of cognitive tests to measure attributes such as working memory. Participants will also provide information about their health and lifestyle, and provide blood samples for extensive analyses, including genetic testing.

There have been many studies into these topics but, unlike COBRA, they have typically compared young adults with older ones, and study cohorts have been small. Probably for these reasons, the results have been contradictory. "These cross-sectional studies have led to discrepancies in the literature," says Bäckman. "Individuals vary massively in how their brains function and the speed of their age-related 
decline." In addition, he points out, comparing a cohort born in the 1930s with one born in the 1980s introduces confounding factors: variables other than age, such as early experiences, environment and education, will be different between the two groups.

Even so, cross-sectional studies have provided some reliable pointers. They have shown that as the brain ages, the grey matter shrinks and the white matter starts to break up. Shrinkage is particularly evident in the prefrontal cortex, where high-level thinking and reasoning takes place, and the hippocampus, which plays a central role in memory. There are fewer receptors and other proteins too, but the most dramatic fall is in the amount of dopamine (see 'Ages of dopamine') - a neurotransmitter whose many functions include movement control, general motivation and learning. From early adulthood onwards, dopamine levels drop by about $10 \%$ per decade, making it a powerful marker for brain ageing.

Some cognitive functions seem to be more sensitive to the ageing process than others. Semantic long-term memory - general knowledge not linked to personal experience - is typically well preserved as we age, but many other attributes start to decline. Reaction speed deteriorates rapidly, for example. Episodic memory, which allows us to reflect on past events, and working memory, which allows distinct pieces of information to be held transiently in the mind, both deteriorate with the advancing years. As a result, as people age they are more easily distracted and find it more difficult to switch quickly between cognitive tasks. They also tend to forget names. And they complain that by the time they open the fridge door they can no longer remember what they wanted - but they still know what a fridge is for.

Not everyone is convinced that some cognitive functions fade faster than others, however. Ulman Lindenberger, a COBRA collaborator and director of the Centre for Lifespan Psychology at the Max Planck Institute for Human Development in Berlin, suspects that all functions fade together within an individual, but that previous studies have been too simple to capture this effect. He hopes that COBRA's approach of using different cognitive tests to measure the same function will help clarify such questions.

\section{A HEALTHY INTEREST}

Long-term, prospective studies such as COBRA tend to be expensive to run and take many years to gather data. Yet they provide the best opportunity to address many important questions about ageing. For example, which structural changes in an individual's brain affect the different cognitive skills? What genetic or environmental factors will be protective? What is the best way to develop treatment strategies?

Most longitudinal studies into ageing focus on dementias such as Alzheimer's disease, which is associated with deposits of amyloid

plaque and tangles of tau protein in the brain. Politicians find it easier to support research on such burdensome diseases than on healthy cognitive ageing, says Bäckman. One of the largest studies of this sort is the Alzheimer's Disease Neuroimaging Initiative (ADNI), based at the University of California, San Francisco (UCSF), and funded by the US National

Some cognitive functions seem to be more sensitive to the ageing process than others.

Institutes of Health and several pharmaceutical companies. It studies 200 people with Alzheimer's disease, 400 subjects with mild cognitive impairment, and 200

elderly controls with no diagnosed mental deterioration. Its primary aim is to find markers that can predict the onset or progression of Alzheimer's, although data from subjects with mild cognitive impairment and controls without dementia will provide leads for understanding non-pathologic ageing.

\section{AGES OF DOPAMINE}

Levels of the neurotransmitter dopamine are higher in some cortical regions in young brains (left) than in old brains (right).

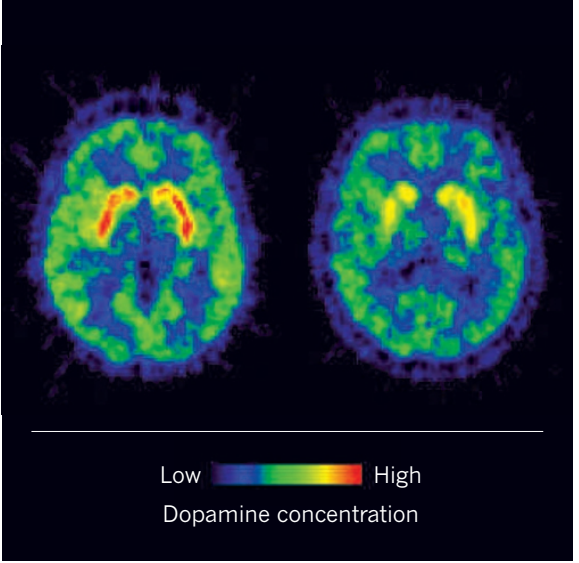

Understanding how Alzheimer's disease begins will also help studies on healthy ageing screen out those with pathologies to ensure that researchers are not detecting the wrong thing. "We are never sure if the cognitive declines we are measuring are actually the otherwiseinvisible beginnings of a dementia that does not become overt during the study," says behavioural neuroscientist Susan Resnick of the US National Institute on Aging in Baltimore, Maryland. Detection of abnormal levels of protein is not a specific enough test. "Nearly a third of old people who are cognitively normal have enough amyloid and tau in their brains to meet the criteria for Alzheimer's disease."

Another problem with longitudinal studies is that it can be hard to convince people to keep returning - a particular problem for ageing studies. "As subjects decline, their families become overstressed and the study seems lower priority," says ADNI director Michael
Weiner at UCSF. In an attempt to avoid this issue, COBRA is recruiting subjects in the northern Swedish town of Umeå, where the population is settled and has strong trust in health authorities.

\section{EXERCISE FOR THE BRAIN}

Describing the process of normal cognitive ageing is a necessary prelude to helping people achieve it. Bäckman believes that successful cognitive ageing depends on maintaining brain integrity - the fewer signs of pathology or brain shrinkage, the better the cognitive function. This corresponds with the widely held view that if we lived long enough, we would all eventually accumulate enough physical damage in the brain to develop dementia. Damage is caused by the accumulation of amyloid plaques or tau tangles, or leakages from small blood vessels in the brain, which occur at different speeds in different individuals. The brain maintenance theory is consistent with evidence that aerobic exercise can slow cognitive decline by stimulating production of brain-derived neurotrophic factor, which helps the growth of new blood vessels, not only in the muscles being exercised but in the brain as well.

But it's a rare octogenarian who can take a long run or play a heart-pounding game of squash, so scientists hope that studies such as COBRA will lead to alternative strategies. One idea is to develop drugs that improve particular types of neurotransmission, but this might have limited value against a background of brain tissue shrinkage. Another strategy could be to use computer-based cognitive training programmes designed to sharpen mental processing speed or working memory. Several studies have shown that people of all ages can improve their performance on specific computer tasks in which they train. Neuroscientist Adam Gazzaley at UCSF is so confident in the potential of this approach that he is developing commercial software for use at home. But even he admits there is no evidence that the improvements seen in computer-based tasks can help with life-related activities — such as helping you remember why you went to the fridge.

As is perhaps appropriate for ageing research, answers will come only with time. "Once we have a tighter link between neurochemistry and structural changes in the brain in relation to cognitive outcome, we'll know what are the main pacemakers of change at this level," says Lindenberger. “Then we'll know how to think about interventions."

The af Jochnick brothers, meanwhile, are learning from the science they support. Both work out in the gym and play tennis. "Our cosmetics helped keep skin young," says 72-yearold Robert. "Hopefully this research will eventually help keep brains young."

Alison Abbott is Nature's senior European correspondent based in Munich. 